\title{
Pulse oximetry recording in children with adenotonsillar hypertrophy: usefulness in the diagnosis of obstructive sleep apnea syndrome
}

\author{
Carlos T. Velasco Suárez M.D..$^{a, c}$, Juan M. Figueroa Turienzo M.D. ${ }^{a, c}$, Fabián Len M.D. ${ }^{b}$ \\ and Enrique Mansilla M.D. ${ }^{b, c}$
}

\begin{abstract}
Introduction. Obstructive sleep apnea syndrome (OSAS) is the most serious consequence of adenotonsillar hypertrophy (ATH). The gold standard diagnostic method is polysomnography (PSG) with nocturnal oximetry, but it requires expensive equipment, the presence of a technician and a specialized doctor, and is very time consuming, making the procedure difficult. The recording of pulse oximetry monitoring during sleep may serve as a diagnostic approach. In pediatrics, few studies have been published, and their results have been controversial. Objective. To establish the diagnostic value of the visual analysis of the recorded nocturnal oximetry monitoring vs. the PSG.

Population. Children with clinical suspicion of OSAS secondary to ATH referred to PSG. Children with other associated diagnoses (myopathy, craniofacial malformations, etc.) were excluded.
\end{abstract}

Methods. 1) A visual analysis (using our own algorithm) of the oximetry recorded simultaneously with the PSG was performed;2) the diagnostic value of the pulse oximetry vs. the PSG was established. Both tests were conducted in a blinded and random fashion by two doctors. Results. A total of 167 PSGs were included; the PSG showed OSAS in 75 children and simple snoring in 92; 65 oximetries were considered pathological and in agreement with the PSG in relation to OSAS; 10 children with mild OSAS in the PSGs had normal oximetries. The recorded pulse oximetry showed a sensitivity of $86.6 \%$ and a specificity of $98.9 \%$ for detecting OSAS. Conclusions. The visual analysis of recorded pulse oximetry monitoring during sleep is highly useful for the diagnosis and management of these patients.

Key words: children; obstructive sleep apnea syndrome; polysomnography, nocturnal oximetry.

http:/ /dx.doi.org/10.5546/aap.2013.196

\section{INTRODUCTION}

Obstructive sleep apnea syndrome (OSAS) is given by the repetitive presence of upper airway obstruction episodes during sleep. Multiple anatomical and functional factors occur simultaneously with OSAS; however, the main triggering factor of this syndrome in children is adenotonsillar hypertrophy (ATH). OSAS causes alterations in different systems and organs which turn into clinical symptoms and disturbances that can lead to major complications. The principal and most common manifestations of OSAS include hyperactivity and neurocognitive disorders, growth retardation, enuresis and systemic or pulmonary arterial hypertension. Reduced oxygen saturation $\left(\mathrm{SaO}_{2}\right)$ caused by airway obstruction episodes is one of the known background conditions for developing OSAS. In most children with ATH and OSAS, an adenotonsillectomy leads to a significant improvement of, or even the recovery from, OSAS..$^{1-4}$

The prevalence of OSAS in children ranges from $2 \%$ to $7 \%$, according to different investigations. Snoring and noisy breathing during sleep are the first warning symptoms, but most children with simple snoring do not suffer from apneas. Simple, non-apneic snoring has a prevalence of around $6-12 \%$ among children., While simple snoring and its impact at a behavioral and cognitive level are the subject of numerous studies, so far there is consensus that it does not constitute per se an indication for an adenotonsillectomy. ${ }^{7,8}$

Therefore, it is essential to establish which children with ATH and nocturnal snoring have OSAS and which, simple snoring, so as to decide on a treatment and the need to initiate it.

Studies have demonstrated that surveys on symptoms administered to the parents of these children have a diagnostic value only in extreme cases of disease or normality, but 
have not proven to be conclusive for most patients. The gold standard diagnostic method is the polysomnography (PSG) with nocturnal oximetry. ${ }^{1-4}$

A PSG requires high cost equipment, the work of a highly specialized technician and doctor, and is very time consuming, making the procedure difficult, especially in the context of a hospital. Even in the private sector, the delays related to the performance of the test and the assessment of results are too long; for this reason, the search for simpler and less expensive methodologies has been a continuous concern.

In adults it is common to use a pulse oximetry monitoring during sleep as an approximate diagnostic method for OSAS. ${ }^{9,10}$ In children pulse oximetry has been sometimes considered an alternative for diagnosis, ${ }^{11}$ but few studies have compared pulse oximetry and PSG, and their results are controversial. ${ }^{12,13}$ The advantages posed by the oximetry are that it requires little equipment (small device, easier to use and with a much lower cost than a polysomnograph), it can be done in the patient's home (thus avoiding inconveniences and hospitalization costs) and its results can be analyzed in an easier and faster manner than those of a PSG. A prospective study was performed to assess pulse oximetry as a diagnostic method in children with ATH and clinically suspected OSAS.

\section{OBJECTIVE}

To establish the diagnostic value of the visual analysis of the recorded nocturnal oximetry monitoring vs. the PSG in a population of children with ATH and suspected OSAS.

\section{POPULATION AND METHODS}

A prospective study was performed and it included children aged 2-16 years, with ATH and clinical suspicion of OSAS (frequent or continuous snoring sometimes associated with breathing pauses or difficulty during sleep, as per the parents' or caregivers' account), referred to CIRES for a PSG in the period between May $1^{\text {st }}, 2005$ and May $1^{\text {st }}, 2009$. Children with other associated diagnosis (myopathy, morbid obesity, craniofacial malformations, encephalopathy, heart conditions, etc.) were excluded.

The study was approved by the Ethics Committee and parents gave their informed consent.

All children underwent a PSG using digital equipment (Akonic Neurotrace) and the following parameters were recorded: EEG, eye movements,
EMG of the mentonian region, monitoring of nasal-oral airflow (thermistor), chest and abdominal movements (piezo sensor belts), ECG, and pulse oximetry (NONIN 8008JFW).

The system software simultaneously records all parameters and then retrieves data for a visual on-screen analysis, jointly or separately. The complete PSG record was retrieved, including oximetry, and the oximetry record alone was also obtained. Both records were analyzed in a blinded and random fashion by two specialized doctors. For the PSG analysis, the screens were watched using 20 second-interval records as per usual standards of sleep stages and other variables. OSAS was considered positive if the PSG showed an apnea-hypopnea index (AHI) equal to or greater than 1 (obstructive apnea: a drop in the airflow signal amplitude equal to or greater than $80 \%$ associated with an effort in chest and abdominal movements; hypopnea: a drop in the airflow signal amplitude of $50-80 \%$ associated with a reduction in $\mathrm{O}_{2}$ saturation of at least $4 \%$ or a micro arousal). ${ }^{14}$

For the pulse oximetry analysis, the entire record was visually analyzed with all the recording collected in just one screen. Artifacts were visually ruled out (a vertical and marked fall in the signal). In this kind of graphic representations, desaturations associated with apnea appear as consecutive falls and rises in the oximetry line, usually grouped in clusters secondary to a chain of multiple obstructive apneas (Figures 1 and 2). Based on a single existing literature reference ${ }^{12}$ and on our personal experience, an oximetry was considered positive if at least two desaturation clusters were observed, with at least one cluster with a fall below $90 \%$.

For the analysis of the sample size, based on prior studies on adults that show that the sensitivity and specificity of the oximetry range between $31 \%$ and $98 \%$, and between $41 \%$ and $100 \%$, respectively, ${ }^{9}$ and on the fact that the percentage of pathological polysomnographies (confirmation of OSAS) in children with clinical suspicion of OSAS and ATH is approximately $40 \%$ in almost all series, it was estimated that to determine the sensitivity and specificity of the test with an $80 \%$ confidence level and a 5\% accuracy 151 patients were required.

\section{Statistical analysis}

Data were presented in a normal distribution using means and standard deviations, and if the distribution deviated from normality, using medians and ranges. 
Sensitivity, specificity, positive and negative predictive values of pulse oximetry vs. PSG were analyzed.

The likelihood ratio that indicates the probability of OSAS occurring post-test was also estimated according to oximetry results.

The discrimination capacity of the oximetry was assessed using the area under the curve (receiver operating characteristic [ROC] curve). The accuracy of the test increases as the area under the curve reaches 1 .

The STATA 9.1 software for Windows (Stata Corp, Texas, 1996) was used for data analysis and the EPIDAT 3.1 software was used for calculating the sample size.

\section{RESULTS}

A total of 175 polysomnographies were performed during the screening period in children with suspicion of OSAS and ATH; 167 met the required criteria. Two patients with myopathy, two with morbid obesity and PraderWillis syndrome, one eight-month old infant and three patients whose PSG were not analyzable due to technical difficulties were excluded.

Out of these 167 patients, 106 (63.5\%) were males. The mean age was 6.75 years old (range: $2-16)$. OSAS was the diagnosis in $45 \%$ of PSGs, and the oximetry was positive in most of them (Tables 1 and 2). Ten children diagnosed with mild OSAS (mean apnea-hypopnea index: 2.02; range: 1.2-4.4) in the PSG had a negative oximetry. The oximetry showed an adequate sensitivity and high specificity for the diagnosis of OSAS (Figure 3).

\section{DISCUSSION}

Up to this date, only two studies that assessed

FIGURE 1A. Polysomnography extract from a patient with a negative result for obstructive sleep apnea syndrome

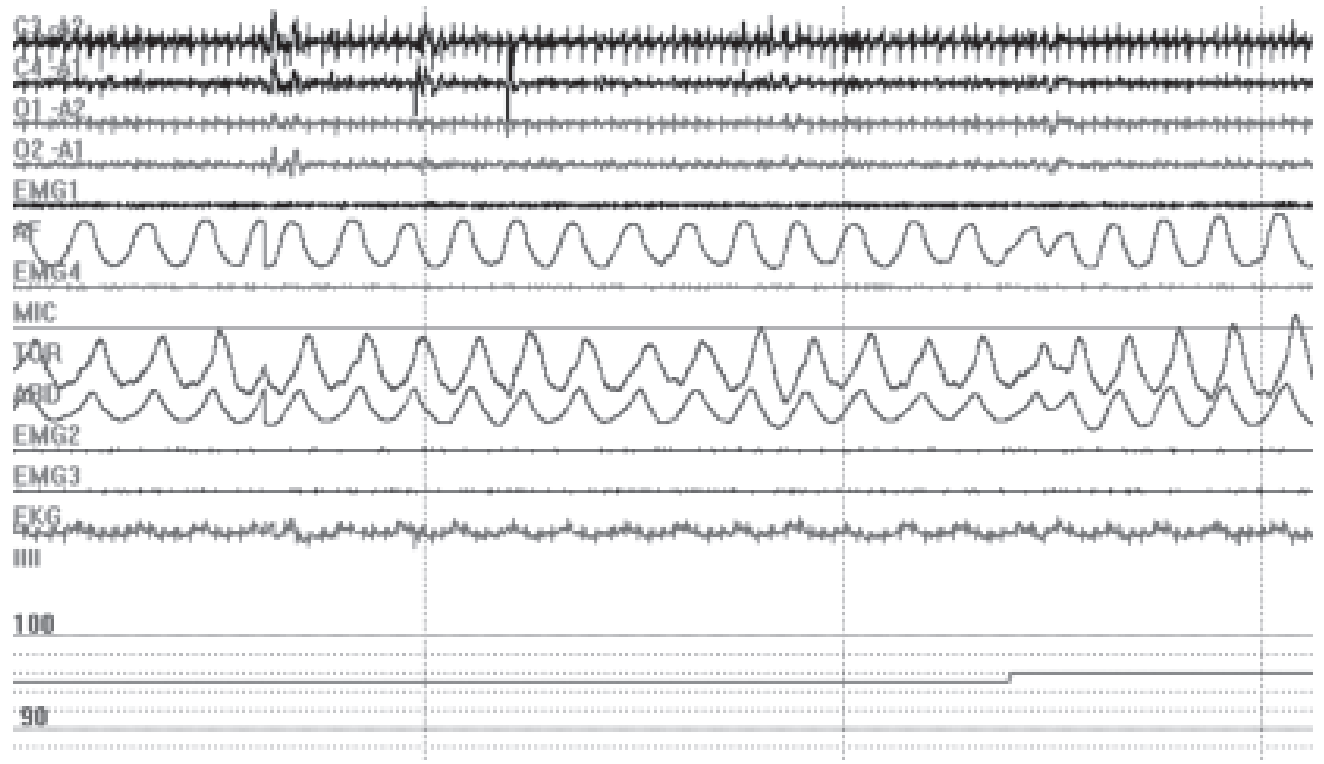

FIGURE 1B. Negative oximetry for obstructive sleep apnea syndrome (same patient)

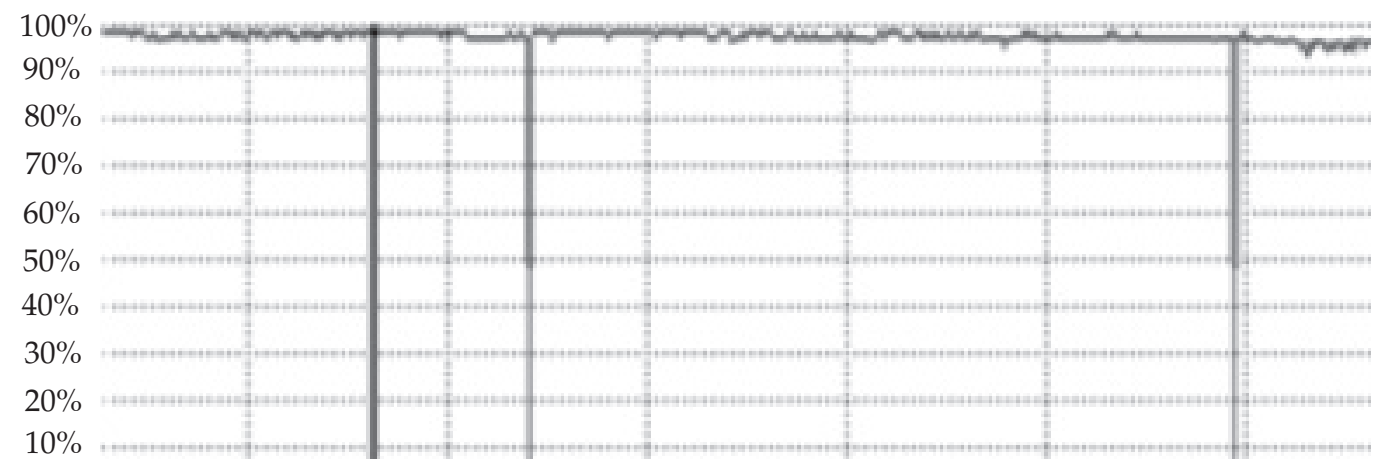


FIGURE 2A. Polysomnography extract from a patient with a positive result for obstructive sleep apnea syndrome

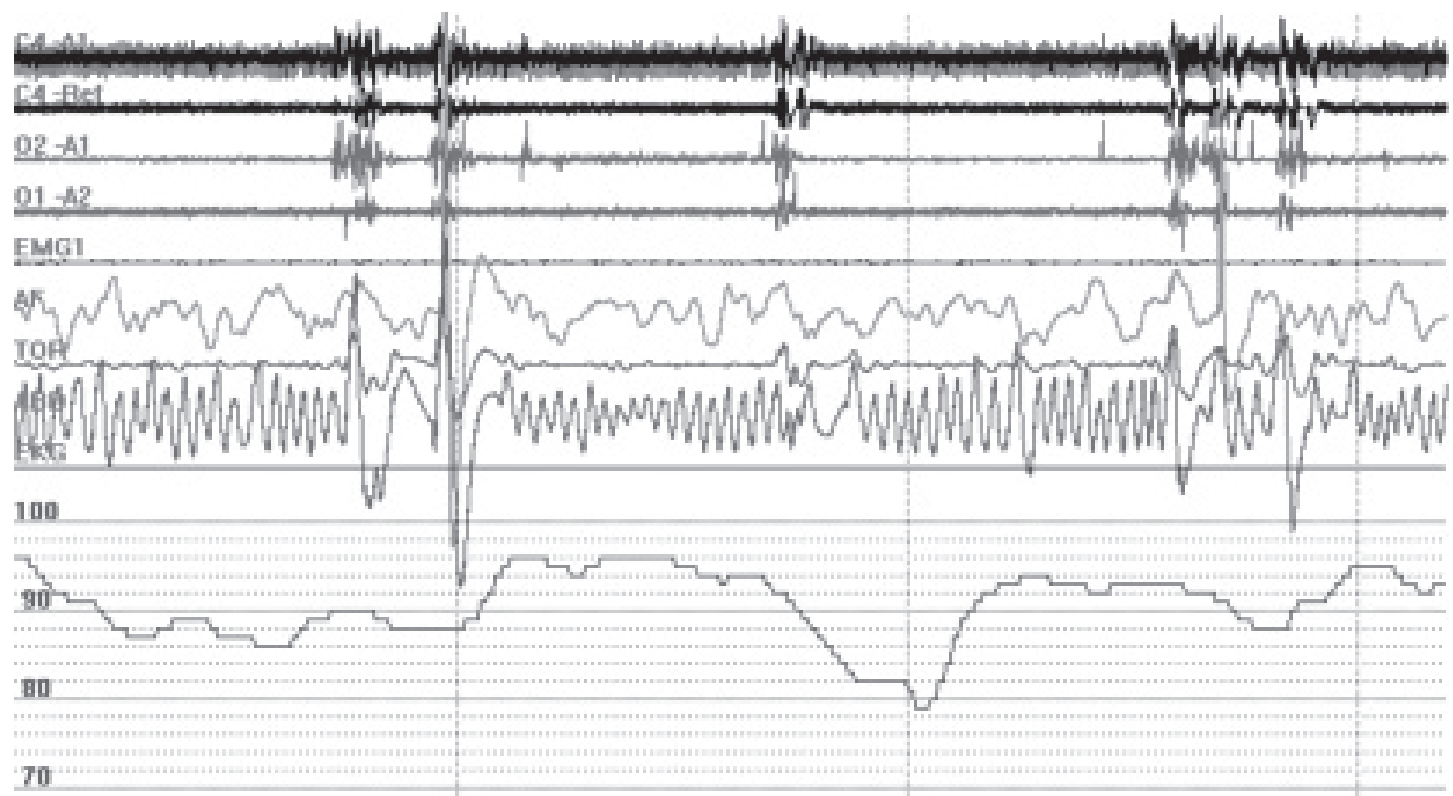

Figure 2B. Positive oximetry for obstructive sleep apnea syndrome (same patient)

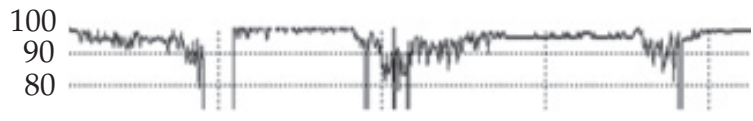

TABLE 1. Oximetry and polysomnography results

\begin{tabular}{lcc}
$(\mathbf{n}=\mathbf{1 6 7})$ & Polysomnography $(+)$ & Polysomnography (-) \\
\hline Oximetry (+) & $65(\mathrm{~T}+)$ & $1(\mathrm{~F}+)$ \\
Oximetry (-) & $10(\mathrm{~F}-)$ & $91(\mathrm{~T}-)$ \\
\hline
\end{tabular}

$\mathrm{T}+$ : true positive; $\mathrm{T}-$ : true negative; $\mathrm{F}+$ : false positive; F-: false negative.

TABLE 2. Oximetry: sensitivity, specificity and predictive value

\begin{tabular}{lcc}
\hline & Value & $\mathbf{9 5 \% ~ C I ~}$ \\
\hline Sensitivity & 0.87 & $0.76-0.93$ \\
Specificity & 0.9891 & $0.94-0.99$ \\
Positive predictive value & 0.98 & $0.92-0.99$ \\
Negative predictive value & 0.901 & $0.82-0.95$ \\
Likelihood ratio (+) & 79.73 & $11.33-61.17$ \\
Likelihood ratio (-) & 0.13 & $0.07-0.24$ \\
\hline
\end{tabular}

Sensitivity calculation $(\mathrm{S})=(\mathrm{T}+) /(\mathrm{T}+)+(\mathrm{F}-)=$

Specificity calculation $(\mathrm{S})=(\mathrm{T}-) /(\mathrm{F}+)+(\mathrm{T}-)=$

Positive predictive value calculation $(\mathrm{PPV})=$

$(\mathrm{T}+) /(\mathrm{T}+)+(\mathrm{F}+)=$

Negative predictive value calculation $(\mathrm{NPV})=$

(T-) / (T-) + (F-) =

( $\mathrm{T}+$ : true positive; $\mathrm{T}-$ : true negative; $\mathrm{F}+$ : false positive;

F-: false negative) the value of oximetry vs. PSG for the diagnosis of OSAS in children have been published. One of the studies did not find an adequate agreement between both diagnostic methods. ${ }^{13}$ In this study, the PSG and the oximetry were done at different times and circumstances (at-home oximetry test, PSG in the lab 48 hours later) and the diagnosis of a normal or abnormal oximetry was obtained using an automatic analysis system included in the oximeter software, with no participation of the

FIGURE 3. ROC curve for the oximetry

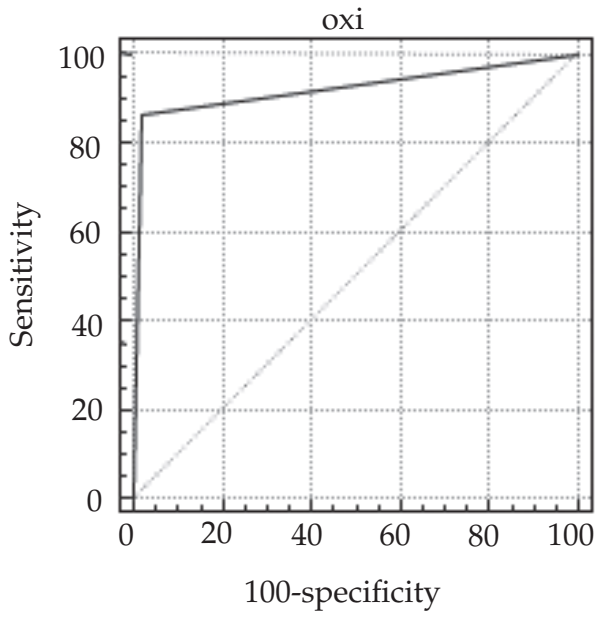

Area under the curve $=0.922$

Standard error $=0.023$

$95 \%$ confidence interval $=0.870-0.958$ 
physician. The calculation made by the software was based on comparisons between consecutive oximetry values and tended to overestimate small variations and to underestimate major variations.

In the other published study, conducted with simultaneous recordings, authors observed an adequate correlation. ${ }^{12}$ Our analysis algorithm was similar to the one used in that study, but in that case authors proposed a higher number of desaturation clusters (three or more) and all drops had to be below $90 \%$.

Based on our preliminary experience, such criteria left an important number of children undiagnosed; therefore, we decided to empirically modify the criteria. A likely cause of such a disagreement can be recording duration, which varied from 8 hours in Brouillette's study to approximately 5 hours in our study. This may lead to a reduced REM sleep duration and, as a consequence, a lower number of total apneas because children with obstructive apneas almost exclusively develop such apneas during REM sleep. The fact that the extrapolation of the number of clusters required per hour of sleep is similar in both algorithms ( 0.4 clusters / hour of sleep) favors such possibility.

The visual analysis of the oximetry record using an adequate oximeter and software is simple and fast (only a couple of minutes) and allowed us to make an accurate diagnosis in most children. Although a hurdle to the visual analysis is that it depends on a trained observer, it should be noted that to this date this is an insurmountable obstacle when it comes to sleeping disorders because even the PSG assessment is observer dependent and there is no consensus regarding the validation of other types of automatic analyses.

Severe hypoxemia (falls below $85 \%$ ) was observed in a significant number of children, and this led to scheduling the adenotonsillectomy for an earlier date. The 10 children with false negative results in the oximetry had mild OSAS (AHI: 1-4) and no hypoxemia (no falls below $90 \%$ recorded throughout the test). Since no desaturations below $90 \%$ occurred, such disparity was expected given the definition adopted for a positive oximetry (at least one fall below $90 \%$ ).

Current knowledge indicates that upper airway obstruction during sleep, even without hypoxemia, may have an impact on the patient's health and quality of life; therefore, it should be reasonable to use a PSG as a tool for a definitive diagnosis before classifying a patient as a "simple snorer." However, the oximetry analysis algorithm proved useful for the management of most of our patients. It should be noted that severity in our population ranged from mild to moderate $(87 \%$ of children had an $\mathrm{AHI}$ between 1 and 10), and this is the most common finding in everyday practice.

The implementation of adequate centers, with good equipment and staff trained for this type of monitoring, would allow to reduce the number of referrals for PSGs and the related costs and, at the same time, to establish the severity of the patient's condition swiftly to make an adequate order of surgery waiting lists.

Lastly, the following study limitations should be underscored: 1) it exclusively focuses on a population of snoring children with ATH and no other associated diseases, so the presence of a lung, neuromuscular or heart condition may negatively affect the validity of our diagnostic approach; 2) although software for analysis and data graphic representations operate similarly in all oximeters, there may be differences in the models and brands that may have an impact, even if small, on the sensitivity and specificity of oximetry vs. PSG.

\section{CONCLUSIONS}

The visual analysis of recorded pulse oximetry monitoring during sleep is highly sensitive and specific in relation to the diagnosis of OSAS in children with ATH and clinically suspected OSAS.

\section{Acknowledgements}

We would like to thank Ricardo Nieto, M.D. and Gimena Donth Laeger, M.D. for their generous and selfless help in the statistical analysis of this study.

\section{REFERENCES}

1. Arens R. Obstructive Sleep Apnea in Childhood. Clinical Features. In Loughlin GM, Carroll JL, Marcus CL, Sleep and breathing in children. A developmental approach. 2000, Marcel Dekker, New York. Pp 575-600.

2. LoughlinGM.ObstructiveSleep Apneain Children. Diagnosis and Management. In Loughlin GM, Carroll JL, Marcus CL,. Sleep and breathing in children. A developmental approach. 2000, Marcel Dekker, New York. Pp 625-50.

3. Ali JN, Stradling JR. Natural history of snoring and sleep disordered breathing in children. In Loughlin GM, Carroll JL, Marcus CL, Sleep and Breathing in Children. A Developmental Approach. 2000, Marcel Dekker, New York. Pp 555-74.

4. Sinha D, Guilleminault C. Sleep disordered breathing in children. Indian J Med Res 2010;131:311-20.

5. Eseverri MV, Noya Pdel V, Mac Lean B, Cipriani SA, Remedi Adel R. Arch Argent Pediatr 2008; 106:231-5.

6. Bonuck KA, Chervin RD, Cole TJ, Emond A, Henderson 
J, Xu L, Freeman K. Prevalence and persistence of sleep disordered breathing symptoms in young children: a 6-year population-based cohort study. Sleep 2011; 34:875-84.

7. O'Brien LM, Mervis CB, Holbrook CR, Bruner JL, Klaus CJ, Rutherford J, Raffield TJ, Gozal D. Neurobehavioral implications of habitual snoring in children. Pediatrics 2004; 114:44-9.

8. Li AM, Au CT, Ho C, Fok TF, Wing YK. Blood pressure is elevated in children with primary snoring. J Pediatr 2009; 155:362-8.

9. Netzer N, Eliasson A, Netzer C, Kristo D. Overnight Pulse oximetry for sleep-disordered breathing in adults. Chest 2001; 120:625-33.

10. Schlosshan D, Elliott MW. Sleep. 3: Clinical presentation and diagnosis of the obstructive sleep apnoea hypopnoea syndrome. Thorax 2004; 59:347-52.

11. Section on pediatric pulmonology and subcommittee on obstructive sleep apnoea syndrome. Obstructive sleep apnea syndrome clinical practice guideline: diagnosis and management. Pediatrics 2002;109;704-12.

12. Brouillette RT, Morielle A, Leimaniis A, Waters K, Luciano R, Ducharme R. Nocturnal pulse oximetry as an abbreviated testing modality for pediatric obstructive sleep apnea. Pediatrics 2000: 105: 405-12.

13. Kirk VG, Bohn SG, Flemons WW, Remmers JE. Comparison of home oximetry monitoring with laboratory polysomnography in children. Chest 2003; 124:1702-08.

14. American Thoracic Society. Standards and indications for cardiopulmonary sleep studies in children. Am J Respir Crit Care Med 1996; 153:866-78. 